\title{
Katja Faulstich
}

\section{Nation-Europa-Welt.}

\section{Identitätsentwürfe vom Mittelalter bis 1800}

Symposion an der Universität Kassel, 15.-17. September 2005

An der Universität Kassel fand vom 15. bis 17. September ein Symposion mit dem Titel „Nation-Europa-Welt. Identitätsentwürfe vom Mittelalter bis 1800 “ statt. ${ }^{1}$ Der Einladung des Fachbereichs Sprach- und Literaturwissenschaften (vertreten durch Claudia Brinker-von der Heyde, Andreas Gardt und Franziska Sick) und des Fachbereichs Gesellschaftswissenschaften (vertreten durch Ingrid Baumgärtner) waren mehr als fünfzig Teilnehmer und Teilnehmerinnen aus dem In- und Ausland gefolgt. Die interdisziplinäre Konzeption der Tagung signalisierte bereits das Vorhaben der Veranstalter und Veranstalterinnen, die wissenschaftliche Analyse von Identitätskonstruktionen als ein Phänomen in den Blick zu rücken, das kulturräumliche und zeitliche Grenzen überschreitet. Was aber ist Identität? Was bezeichnen Begriffe wie Nation, Europa und Welt? Wie muss eine wissenschaftliche Auseinandersetzung mit diesen sozialen, kulturellen und politischen Konstruktionen beschaffen sein, die den Prozessen ihrer Konstruktion nachgeht und gleichzeitig ihren ontologischen Status, ihre Universalität und ihre Ursprungsmythen in Frage stellt? Aufgrund der Ambiguität dieser Begriffe und um der Gefahr einer erneuten Zuschreibung zu entgehen, standen methodisch-theoretische Überlegungen im Mittelpunkt des ersten Veranstaltungstages, während der zweite Abschnitt der Tagung vorrangig empirischen Analysen gewidmet war.

Auf die besondere Problematik von identitätskonstruierenden Begriffen verwies Claudia Brinker-von der Heyde (Kassel) in ihrer einleitenden Erläuterung der Konzeption der Tagung. Begriffe wie Nation, Europa und Welt seien keineswegs ontologische Größen, sondern jeweils ethnisch, sozial, räumlich, kulturell und/oder politisch determinierte Konstrukte. Eine interdisziplinär angelegte Begriffs-, Ideen- und Kulturgeschichte dieser Einheiten und ihrer jeweiligen Bezugsgrößen wie Sprache und Literatur erweise sich aber ebenso als ein Desiderat wie die Erforschung der Konstruktion kollektiver Identitäten in der Zeit vom Mittelalter bis 1800. Ziel der Tagung sei es deshalb, die verschiedenen historisch-kulturellen Ausprägungen der Identitätsdiskurse und ihre Argumentationsmuster zu untersuchen. Eine Beschäftigung mit den Prozessen der Identitätsbildung dürfe aber nicht bei dem jeweils Spezifischen der Identitätsentwürfe stehen bleiben, sondern müsse die Gemeinsamkeiten der

$1 \quad$ Der Tagungsband ist soeben erschienen: Ingrid Baumgärtner, Claudia Brinker-von der Heyde, Andreas Gardt, Franziska Sitz: Nation-Europa-Welt. Identitätsentwürfe vom Mittelalter bis 1800. Frabkfurt 2007 (=Zeitsprünge. Forschungen zur Frühen Neuzeit. Band 11, Heft 3/4). 
Kategorisierungen und Argumentationen aufzeigen. Diese Rekonstruktion sei genuine Aufgabe der Geisteswissenschaften.

Jürgen Trabant (Berlin) eröffnete den methodisch-theoretisch orientierten Tagungsabschnitt mit einem Beitrag zur identitätsstiftenden Funktion von Sprache. Er unterschied zwei Identifikationsmodelle, deren gemeinsame Voraussetzung aber die Erfahrung sprachlicher Alterität sei. Das erste Modell entwickelte Trabant auf der Grundlage des ersten Buchs von Dantes „Convivio“. Als einem in der Volkssprache dichtenden lateinischen doctor sei Dante die Diglossie zwischen Volkssprache und Latein zum Problem geworden, die den mittelalterlichen Gelehrten noch keine Schwierigkeit bereitet habe. Als Ursache hierfür benannte Trabant Dantes Programm der Vermittlung von Wissen an das Volk (nobile gente), d. h. an die wohlhabenden Schichten, in der Volkssprache (volgare). In Form der italica loquela sei diese bei Dante zu verstehen als „Sprache der Eltern“ und als Ausdruck der verwandtschaftlichen Nähe zu seinem Volk (gens). Der mit dem Namen italica loquela konstruierte gesellschaftliche und ethnische Raum sei wegen der fehlenden staatlichen Strukturen primär sprachlich und nicht politisch determiniert. Während Dante Sprache in der Tradition Aristoteles' als etwas rein Lautliches (phonè) betrachte, beruhe das seit dem 16. Jahrhundert entstandene, zweite Identifikationsmuster auf der Vorstellung, dass die Sprache eine ganz besondere Form des Denkens sei (vgl. Humboldts Konzept der Weltansicht). Dieses Identifikationsmuster bilde sowohl die Grundlage der modernen Bemühungen um die Beschreibung der Sprachen als auch den Kern des sprachlichen Nationalismus. Die politischen Implikationen dieses Modells und einen möglichen Abschied vom sprachidentitären Diskurs thematisierte der Referent am Ende seines Vortrages. In der Gegenwart stünden sich zwei konkurrierende Modelle gegenüber: ein optimistisches Modell, das von einer frei wählbaren Sprachidentität ausgehe (Coulmas) und ein tragisches Modell (Derrida), das die Täuschungen des Identitären betone und von der schmerzhaften Erfahrung des Sprachverlustes - etwa durch Exil und Unterdrückung - ausgehe.

Einen möglichen Zugang zur Erforschung kollektiver Identitäten stellte Fritz Hermanns (Heidelberg) in seinem Vortrag „Identitätsgeschichte als Mentalitätsgeschichte" vor. Er definierte Identität als Gesamtheit jeweils relevanter Eigenschaften eines Menschen oder einer sozialen Gruppe, wobei diese Eigenschaften sowohl real als auch vorgestellt sein könnten und ihre Relevanz jeweils an den Kontext gebunden sei. Darüber hinaus seien selbst Spezialidentitäten eher als Summe von Einzeleigenschaften, denn als geschlossene Einheiten zu betrachten. Das Konzept deutsche Identität sei in diesem Sinne zu verstehen als die Eigenschaft, die deutsche Staatsbürgerschaft zu besitzen, deutscher Abstammung zu sein, deutsch zu sprechen usw. Für die Identitätsforschung formulierte der Vortragende folgende Leitfragen: Welche Relevanzen kamen den von ihr beschriebenen Identitäten zu? Wo und wann, in welchen Kontexten, kamen sie zum Tragen? Als mögliche Forschungsgegenstände wurden Größen wie die soziale Identität, die Selbst- und Fremdidentität oder die Wunsch- und Angstidentität genannt. Im zweiten Abschnitt stand der Zusammenhang von Identität und Mentalität im Vordergrund, wobei letztere im Sinne der Mentalitätsgeschichte definiert wurde als Gesamtheit aller usuellen Vorstellungen und Einstellungen in einer sozialen Gruppe. Das habituelle Denken, Fühlen, Wollen einer einzelnen Person sei für eine Identitätsgeschichte als Mentalitätsgeschichte aber nur dann von Interesse, wenn es repräsentativ sei für Mentalitäten von gesellschaftlichen Gruppen. Zum Abschluss seines Vortrages illustrierte der Referent seinen Gedanken, dass Identitätsgeschichte 
zugleich immer auch Mentalitätsgeschichte sei (und umgekehrt), anhand des Gedichts „Des Deutschen Vaterland“ von Ernst Moritz Arndt.

Mit den erkenntnistheoretischen Problemen einer Re-Konstruktion kollektiver Identitäten beschäftigte sich Olaf Asbach (Hamburg / FEA Potsdam / Augsburg) am Beispiel der Konstruktionen einer politischen Identität Europas. Identitätskategorien sind nach Asbach Reflexions- und Deutungsbegriffe im Rahmen historischer und sozialer Entwicklungen, in denen sie quasi gegenstandskonstitutive Wirkung entfalten. Eine Rekonstruktion Europas als Identitätskonzept müsse vor allem vermeiden, ihrerseits einen Ursprungsmythos zu konstruieren und Europa als Telos, Subjekt oder Substanz der Geschichte zu präsentieren. Asbach skizzierte zunächst eine Reihe von Versuchen, Europa als politische Idee und als historisch-kulturelle Einheit, d. h. als eine Gemeinschaft mit gemeinsamen Erfahrungen, Werten, Lebensformen und Traditionen $\mathrm{zu}$ rekonstruieren. Er führte aus, dass ein „identifikatorischer Zugang“ (Michael Mitterauer) zur Geschichte Europas seinerseits einen Ursprungsmythos der politischen und kulturellen Einheit Europas konstituiere und legitimiere. Vor dem Hintergrund der dargelegten Problematik entwickelte der Referent das Programm einer historisch-kritischen Erforschung der Entstehung und des Funktionierens europäischer Identitätskonstruktionen, das sich von identifikatorischen Europavorstellungen löst und sich der politischen Instrumentalisierung verschließt. Seine Überlegungen erfuhren im Plenum breite Zustimmung, wenngleich die Absage selbst an ,positive' identifikatorische Europakonzepte kritisch diskutiert wurde.

Im zweiten Abschnitt des Symposions standen empirische Analysen im Mittelpunkt. Zunächst befasste sich Volker Scior (Osnabrück) mit Identitätsmustern historiographischer Texte des Früh- und Hochmittelalters, d. h. mit Identitätsentwürfen gebildeter, schriftkundiger Kleriker wie Adam von Bremen. In den mittelalterlichen Texten der ostfränkischen Chronisten lassen sich nach Scior drei Typen von Identitätsmustern erkennen: eine religiöse, christliche Identität, die sich von den Heiden oder Barbaren abgrenze, eine institutionelle Identität (beispielsweise in Bezug auf ein Bistum) und eine ethnische und politische Identität im vormodernen Sinne. Scior unterstrich die Ambiguität und das weite Bedeutungsspektrum von Begriffen wie gens oder natio im Mittelalter, denen in der Forschung zur frühmittelalterlichen Ethnogenese eine besondere Bedeutung zukomme. Diese Konzepte seien aber vorrangig im Sinne von Stamm oder als politisches Gebilde zu verstehen und nicht als Indikatoren eines frühen Nationalbewusstseins zu interpretieren. Gerade die jüngere Ethnogeneseforschung betone den Status der Völker des frühen Mittelalters als stets im Wandel begriffene Völkergemische, die sich zum Beispiel durch Herkunftsmythen eine eigene Tradition gaben und auf der politisch-realen Ebene durch langjährige Herrschaft zusammenwuchsen. Der Sprachverwandtschaft komme hingegen keine tragende Funktion bei der mittelalterlichen Identitätsbildung zu. Scior kam zu dem Ergebnis, dass Europa im Mittelalter weder eine Rolle als geographische bzw. historische Einheit noch als Wertegemeinschaft gespielt habe. In der anschließenden Diskussion wurde die Schwierigkeit geäußert, von einem individuellen Autor auf zeitgenössische Identitäten zu schließen und das Problem der Autorschaft im Mittelalter diskutiert. Außerdem wurde auf sprachidentitäre Aspekte des Annoliedes verwiesen.

Folker Reichert (Stuttgart) zeichnete ein Bild von „Marco Polos Identitäten“ und wandte sich den Identitäten einer historischen Einzelperson zu. Er entfaltete Marco Polos religiöse Identität als Christ, seine kulturell-territoriale Identität als 
norditalienischer Sohn eines Kaufmanns, seine Identität als „Mann des Großkhans“ und schließlich seine Identität als ,enkulturierter Mongole“. Die letztgenannte Identität markiere insbesondere Marco Polos markante Zwischenstellung zwischen der dominierenden mongolischen Kultur und der Kultur der Nordchinesen. Im Plenum wurden die Zuschreibungsmechanismen der Historiographie deutlich, die zur Stilisierung Marco Polos zum Vorläufer einer präimperialistischen Tradition und zur Marginalisierung des Phänomens der Enkulturation geführt haben. Die Wechselseitigkeit von Kulturkontakten untersuchte Renate Dürr (Frankfurt am Main / Kassel) in ihrem Vortrag über die Jesuiten und Guaraní in den Reduktionen von Paraguay (1609-1768). Sie kam damit auf einen besonders wichtigen Aspekt der Identitätsforschung zu sprechen, die gegenseitige Einflussnahme von Selbst- und Fremdbildern. Dürr definierte Identität als Teil eines Kommunikationsprozesses, deren explizite Bestandteile in Form der nach außen getragenen Selbstwahrnehmung erkennbar seien und deren implizite Bestandteile sich in bestimmten Handlungen oder Strukturen einer Gemeinschaft zeigen. Als Quellen zog sie u. a. Reiseberichte und Berichte der Jesuiten über die Organisation der Reduktionen als „dörfliche Gemeinwirtschaft“ heran, wobei sie unter Verweis auf einige Fallstudien ausdrücklich auf problematische, gattungsimmanente Vorgaben der verschiedenen Textgattungen verwies. Die Referentin hob besonders die Akkulturation des Christentums und die aktive Aneignung der Reduktionen durch die Guaraní hervor. In der Diskussion wurde ihre Bestimmung von Identität als Teil eines Kommunikationsprozesses einhellig befürwortet. Aufgeworfen wurde darüber hinaus die Frage der schwierigen - und aufgrund der Quellenlage nur bedingt möglichen - Rekonstruktion des Blicks der Guaraní auf die Europäer.

Wie und wieweit Identifikation durch räumlich-geographische Zuordnung funktioniert, führte Hartmut Kugler (Erlangen) am Beispiel der mittelalterlichen und frühneuzeitlichen Kartographie aus, wobei er auf das Konzept des „Mental mappings" der Kognitionswissenschaft rekurrierte. In diesem Verständnis sind Karten ein Medium der Wissensorganisation. Wie eine solche Wissensorganisation sich kartographisch manifestiert, erläuterte Kugler anhand von zwei sehr unterschiedlichen Identifikationsmustern. Das erste Muster finde sich im „Liber Floridus“ aus dem 12. Jahrhundert von Lambert von Saint-Omer. In der foliogroßen Weltkarte des „Liber Floridus" ist die Welt durch das T-O-Schema als eine Form der Wissensspeicherung dreigeteilt in die Kontinente Afrika, Asien und Europa. In dieser Karte und der dazugehörigen Ausschnittsvergrößerung Europas sei das primäre Ziel der Darstellung, die Kohärenz der frühscholastischen, christlichen Welt- und Wissensordnung abzubilden. Karthographisch sei dies ablesbar an der Symmetrie und der Gleichförmigkeit der Gebiete, Städte und Regionen. Diese Stilisierung verzerre trotz der zeitgenössischen Kenntnisse die geographischen Verhältnisse bis zur Unkenntlichkeit. Während der eigene Betrachterstandpunkt bei diesem Muster völlig im Gesamtbild aufgehe, mache der Nürnberger Kartograph Erhard Etzlaub diesen zum Ausgangspunkt seiner Kartographie. Etzlaubs Karten wie etwa die berühmte „Romwegkarte“ und die „Reichsstraßenkarte“ seien der Kohärenz der zeitgenössischen Messkunst verpflichtet. Der Betrachter könne mit den Karten die tatsächliche Entfernung und damit die Erreichbarkeit lokaler Einheiten (Städte, Regionen usw.) feststellen.

Klaus Garber (Osnabrück) präsentierte eine Sammlung von Forschungshypothesen, um den spezifischen Beitrag der Literatur zur Herausbildung eines Nationalbewusstseins deutlich zu machen. Seine historische Betrachtung des Verhältnisses 
von Nation und Literatur im Europa der Frühen Neuzeit setzte mit der Volkssprachenproblematik in Dantes „De vulgari eloquentia“ ein und schloss die Dimension der Erfahrung von Krisen als wesentliches Moment der Nationenbildung mit ein. Im Volgare habe Dante eine von allen Partikularismen gereinigte italienische Sprache gesehen, die in Form eines auf die Italiener begrenzten Idioms und eines allen Schichten verständlichen Mediums als erste Nationalsprache konzipiert gewesen sei. Den im Vergleich zur französischen und italienischen Entwicklung verzögerten Übergang der deutschen Literatur zum nationalliterarischen Diskurs datierte der Vortragende auf den Beginn des 17. Jahrhunderts. Der literarische Diskurs dieser Zeit habe viel intensiver an den Errungenschaften des transkonfessionellen und nationalpolitischen Denkens um 1600 partizipiert, als in der Forschung bislang berücksichtigt worden sei. Garber plädierte insgesamt für eine stärkere Gewichtung der europäischen Dimension der Literaturgeschichtsschreibung, die die nationalphilologischen Beschränkungen aufgeben solle.

Mit der „Entdeckung und Gestaltung europäischer Identität in der deutschen Literatur der Frühen Neuzeit" beschäftigte sich Peter Hanenberg (Viseu). Wie schon bei Klaus Garber gerieten damit verstärkt literarische Texte in den Blick. Zunächst führte der Referent das Konzept der Europäisierung aus, unter das er die vielfältigen und divergierenden Prozesse subsumierte, die vom europäischen Kontext ausgehen und die ihn als Ganzen prägen wie etwa die Christianisierung, die Profanisierung und der Nationalismus. Dieser Europäisierung ging der Referent in Grimmelshausens "Simplicissimus" nach sowie in Johann Gottfried Schnabels Roman "Wunderliche Fata einiger See-Fahrer“ und Christoph Martin Wielands „Der goldene Spiegel“. Die Spezifik der Europakonstruktionen leitete Hanenberg von dem jeweiligen historischen Wissensrahmen ab, in dem er die Texte verortete. So müsse Schnabel Europa in Form einer Utopie präsentieren und an einen unbestimmten Ort versetzen, um sein Programm der Verbesserung der Verhältnisse, wie es in den Begriffen Wabrhaftigkeit, Woblfahrt und Nütəlichkeit zum Ausdruck komme, zu entwickeln. Eine Ablösung von den Utopien als Ausdruck einer christlichen Heilslehre erfolge mit der Aufklärung, in der sich eine umfassende Profanisierung des Europabegriffs vollziehe. In dem idealen Staat, den Wieland entwerfe, trete die Logik des Staates an die Stelle der theologischen Ordnung. Gemeinsam sei den unterschiedlichen Europakonstruktionen der behandelten Texte allerdings die Imagination eines jeweils ,anderen Europas".

Andreas Gardt (Kassel) stellte eine historisch-systematische Analyse der Bezeichnungen Nation und Volk auf der Basis von Texten des 17. und 18. Jahrhunderts vor. Das zugrunde liegende Quellencorpus umfasst sprachreflexive Texte unterschiedlicher Art, von zeitgenössischen Grammatiken, Rhetoriken und Wörterbüchern bis zu sprachtheoretischen Abhandlungen oder der Korrespondenz der Mitglieder der barocken Sprachgesellschaften. Die Ausdrücke Nation und Volk wurden im Kontext ihres konkreten Textvorkommens untersucht, d. h. unter Berücksichtigung ihres unmittelbaren semantischen Umfelds und ihrer Verwendung in diskursspezifischen Argumentationsformen. Gardt betonte vor allem die gegenstandskonstituierende Funktion von Sprache, die in zahlreichen Tagungsbeiträgen und Diskussionen wiederholt thematisiert wurde. In der Diskussion wurde insbesondere die Frage der Korpuszusammenstellung diskutiert. Im Sinne einer europäischen Sprachund Literaturgeschichtsschreibung, die während der Tagung mehrfach eingefordert 
wurde, stellte sich die Frage nach einer Erweiterung des Korpus um zentrale Texte beispielsweise der italienischen und französischen Tradition.

Kirsten Mahlke (Konstanz) befasste sich in ihrem Vortrag mit Identitätskonzepten in historiographischen, juristischen und literarischen Texten im frühneuzeitlichen Frankreich. Am Beispiel der „Antiquates“ des italienischen Mönchs Annius von Viterbo, die um 1511 von Jean Lemaire de Belges ins Französische übersetzt wurden, rekonstruierte sie die Entstehung der französischen Nationalidee im 16. Jahrhundert. Der Mythos der gallischen Herkunft sei von den frühneuzeitlichen juristischen Gelehrten genutzt worden, um in einer politischen Krisenzeit die Herleitung des Königshauses von den Trojanern bis hin zu Gomerus alias Gallus, dem Enkel des Überlebenden der Sintflut, zu präsentieren und damit eine Ahnenreihe bis hin zu den Enkeln Noahs zu behaupten. Unter Hinzuziehung von Schriften wie Joachim du Bellays „Deffence et Illustration de la langue françoyse“ (1552) wurde darüber hinaus die Rolle der Sprache als identitätsstiftendes Merkmal im zeitgenössischen Diskurs hervorgehoben. In der Diskussion wurden Parallelen der Argumentationsmuster der französischen und der deutschen Gelehrten des 17. Jahrhunderts aufgezeigt und die Ähnlichkeiten europäischer Ursprungsmythen unterstrichen.

Jürgen Schiewe (Greifswald) zeichnete in seinem Vortrag „Identitätskonstruktionen um 1800. Deutsche Diskurse zwischen Revolution und Restauration" Versuche der Identitätsstiftung durch Sprache bei Gottfried Wilhelm Leibniz, Joachim Heinrich Campe, Ernst Moritz Arndt und Carl Gustav Jochmann nach. Das nationalpädagogische Programm, das Leibniz vorlegt habe, ziele ebenso wie das gescheiterte Sprachreinigungsprogramm Campes auf eine Veränderung der sprachlichen und kommunikativen Verhältnisse in Deutschland. Der Vortragende verwies an dieser Stelle auf das zentrale Anliegen beider Gelehrten: die Ausbildung des Deutschen zu einer allgemein verständlichen, polyfunktionalen deutschen Sprache. Campe habe darüber hinaus das realpolitische Ziel vertreten, die unteren Stände in verfassungsmäßige Rechte zu setzen. Während Leibniz und Campe die sprachlichen und kommunikativen Verhältnisse in Frankreich sowohl als Vorbild als auch als Kontrastfolie genutzt hätten, zeige der Franzosenhass Arndts die Gefahr der nationalistischen Indienstnahme von Identitätskonstruktionen, die auf Sprache aufbauen. Im letzten Teil seines Beitrags plädierte Schiewe deshalb in Anlehnung an Jochmann dafür, Identität nicht an die Sprache per se zu binden, sondern an den Kommunikationsgebrauch bzw. an konkrete Kommunikationsformen.

Die Schlussdiskussion hatte vorrangig zum Ziel, mögliche Forschungsschwerpunkte $\mathrm{zu}$ benennen und mögliche Forschungskooperationen zu ermitteln. Andreas Gardt verwies auf drei zentrale Ausweitungen der in der Tagung theoretisch, methodisch und empirisch umrissenen Identitätsforschung: Die Trias „Nation-Europa-Welt“ solle in den Einzelanalysen und in den Anschlussprojekten zusammengeführt werden, dabei solle zweitens der nationalphilologische Rahmen gesprengt und ein interdisziplinärer Zugang gewählt werden, der drittens den Untersuchungszeitraum vom Mittelalter bis 1800 umspannen solle. Ingo Warnke (Kassel) wies auf eine Erweiterungsmöglichkeit des Projektes um eine transnationale Komponente hin, beispielsweise in Form einer Analyse des Menschenrechtsdiskurses. Ebenso thematisiert wurden die stärkere Gewichtung des Faktors Raum und der Ethnologie (Folker Reichert) sowie der Zeit nach 1800 (Kirsten Mahlke). Große Zustimmung erfuhr die Forderung, die nationalphilologischen Grenzen zu überwinden.

Das weite Feld der theoretischen wie methodologischen Fragestellungen und das 
zeitlich, räumlich-kulturell wie auch sprachlich weit verzweigte Forschungsfeld wurde durch die gründliche Organisation und Konzeption der Tagung umklammert und in den Einzelbeiträgen sorgfältig abgesteckt, sodass sich der Disziplinen, Einzelsprachen und Epochen übergreifende Ansatz sowohl in den Diskussionen als auch in den anregenden Gesprächen im Verlauf des Rahmenprogramms als überaus fruchtbar erwies.

Adresse der Berichterstatterin:

Katja Faulstich, Universität Kassel, Fachbereich 02 - Sprach- und Literaturwissenschaften, Institut für Germanistik, D-34109 Kassel.

E-mail:faulstich@uni-kassel.de 